\title{
Cabanis und die Gewißheit der Heilkunde ${ }^{1}$
}

\author{
Von Erna Lesky, Wien
}

1

Die Fragen, die sich mit den denkmethodischen Grundlagen der Medizin beschäftigen, fanden ihre früheste Formulierung dort, wo die Heilkunde als Wissenschaft geboren wurde: im Griechenland des 5. Jahrhunderts v. Chr. Hier handelt es sich vor allem um zwei Schriften des hippokratischen

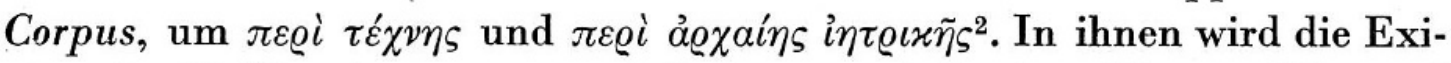
stenz der Heilkunde gegen mannigfache gegnerische Angriffe verteidigt und dabei der Beweis versucht, daß die Medizin über wohlgegründete Methoden verfüge.

Schon in dieser ersten Auseinandersetzung im letzten Drittel des 5. Jahrhunderts ${ }^{3}$ wurden aus Angriff und Verteidigung bestimmte Einsichten in die Verfahrensart der Heilkunde gewonnen, die für jede folgende Erörterung des Problems grundlegend werden sollten. Dies konnte nur erfolgen, weil bereits die erste Verhandlung dieser Fragen, die im Geiste der griechischen Aufklärung vor sich ging, Aussagen zeitigte, die Grundphänomene ärztlichen Denkens und Handelns angingen.

Welches sind nun die Angriffe, in deren Widerlegung die hippokratische Medizin ihre Methoden enthüllte und damit zugleich ihre Existenz bewies? Es sind die seither so oft erhobenen: Heilen Krankheiten nicht auch durch Zufall (de prisc. med. c. 1. 36, 12; de arte c. 4. 11, 5 ff.), heilen sie nicht auch von selbst ohne Arzt (de arte c. 5. 11, 20 ff.) ? Und was ist das für eine Kunst, die behauptet, Krankheiten heilen zu können, wenn sie doch selbst so viele Krankheiten als unheilbar erklären muß (de arte c.8.14,1 ff.)? Diese Vorwürfe vor allem sind es, an denen die junge Heilkunde ihre Methoden überprüft.

Sie nimmt Stellung zur Frage des Zufalls, indem sie auf die Allgemeingültigkeit des Kausalgesetzes verweist (de arte c. $6.13,1 \mathrm{ff} .=\mathrm{G} .43 \mathrm{f}$.) :

\footnotetext{
${ }^{1}$ Dieser Aufsatz stellt die Ausarbeitung eines Vortrages dar, der am 28. September 1954 auf der Tagung der Deutschen Vereinigung für Geschichte der Medizin, Naturwissenschaft und Technik in Sigmaringen gehalten wurde.

2 Die beiden Schriften werden im folgenden nach Seiten und Zeilen der Ausgabe J.L. HeIBERGs im Corpus Medicorum Graecorum vol. I/1, Berlin 1927, zitiert. Dazu vgl. man die Ausgaben und Kommentare von Тн. Gomperz, Die Apologie der Heilkunst, Leipzig 1910, für de arte, dessen Übersetzung im folgenden benutzt wurde (zitiert als G. mit Seitenzahl) ; für de prisc. med. A.J.Festugière, Hippocrate. L'ancienne médecine, Paris 1948.

${ }^{3}$ A. J. Festugière, a. O. XVIII.
} 
«Denn bei allem, was da geschieht, kann man finden, daß es durch etwas geschieht; ... die Heilkunst aber hat in dem, was durch etwas geschieht und was sich vorhersehen läßt, ihr Bestehen und wird es darin allezeit haben.»

Auf den festen Zusammenhang von Ursache und Wirkung, auf die Ordnung der Natur, gründet also die Heilkunde ihre Prognose und damit ihr Handeln am Krankenbett. Denn Prognose ist im Sinne der hippokratischen Medizin mehr als bloße Voraussicht des Krankheitsverlaufes und Krankheitsausganges; Prognose ist gleichzeitig auch Voraussetzung für die Aufstellung eines Heilplanes, also Voraussetzung jeder ärztlichen Therapie ${ }^{4}$. Indem die Heilkunde heilsame Mittel von schädlich wirkenden zu trennen und richtig anzuwenden vermag, weiß sie dieses ihr Tun von den in der Natur selbst liegenden Gesetzlichkeiten abhängig und damit ihre Existenz und ihr Verfahren begründet (de arte c. $5.12,1 \mathrm{ff}$.).

Im 8. Kap. von de arte $(14,1 \mathrm{ff}$.) versucht der Verfasser die Heilkunde gegen den Vorwurf in Schutz zu nehmen, daß sie zu Unrecht nicht auch die unheilbaren Krankheiten behandle. Dabei geht er auf die dem Arzte zur Verfügung stehenden Kenntnismittel - der Verfasser nennt sie im handwerklichen Denken der Griechen «Werkzeuge» $(14,12)$ der Heilkunst - ein. Er unterscheidet die unmittelbare Wahrnehmung durch die Sinne, mit Hilfe deren man an einem äußeren, sichtbaren Leiden feststellt (de arte c.9.15, $8 \mathrm{ff}$. = G. 49), «was an ihm hart und was an ihm weich, und was warm und was kalt ist, und durch welcher Dinge Anwesenheit oder Abwesenheit es jedesmal eines von diesen ist.» Viel schwieriger als bei den äußeren, sichtbaren Krankheiten gestaltet sich die Erkennung innerer, verborgener Leiden (de arte c. 11.16,20 ff. = G.53) : «Denn da jener (sc. der Arzt) das Übel nicht mit Augen schauen konnte und nicht mit den Ohren vernehmen, so verfolgt er es durch Schlüsse.» Es tritt also zu der (vom Autor) rein sinnlich vorgestellten Wahrnehmung ergänzend jenes Verfahren hinzu, das aus den sichtbaren Zeichen das unsichtbare Krankheitsgeschehen zu erschließen

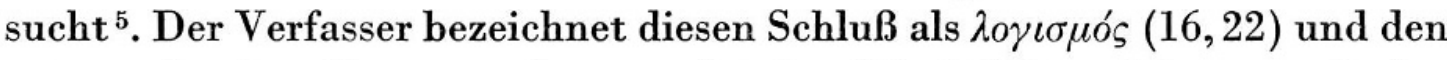
entsprechenden Vorgang als $\tau \varepsilon x \mu \alpha i \varrho \varepsilon \sigma \vartheta \alpha \iota(18,9.18)$ und hat so mit den

4 Th. Meyer-Steineg, Die Bedeutung der Prognose in den hippokratischen Schriften, Arch.

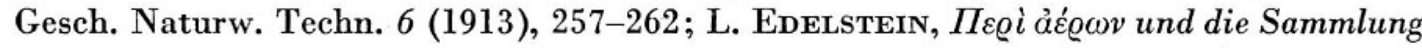

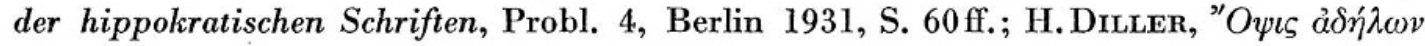
$\tau \dot{\alpha}$ qaıvó $\mu \varepsilon v a$, Hermes 67 (1932), 20f.; W. Nestre, Griechische Studien, Stuttgart 1948, S. 530 .

${ }^{5}$ Zur Stelle H. Diller, a. O.20f.; W. Nestle, Vom Mythos zum Logos, Stuttgart 1940, S. 304 . 
seiner Zeit gegebenen Mitteln ein Grundphänomen ärztlicher und überhaupt naturwissenschaftlicher Erkenntnisfindung eindringlich dargestellt ${ }^{6}$. Aber er nennt auch noch ein anderes Verfahren außer den bereits genannten (de arte c. 12.18, $10 \mathrm{ff} .=\mathrm{G} .57)$ : «Wenn aber all dies nichts von sich selber verrät und die Natur nichts freiwillig entsendet, so hat die Kunst einen Folterzwang ersonnen, durch welchen die Natur, mit unschädlicher Gewalt genötigt, etwas von sich gibt; indem sie es aber abgab, zeigt sie denen, die die Kunst verstehen, was zu tun ist.» Um nichts anderes als um das Experiment als zusätzliches Erkenntnismittel ärztlicher Forschung handelt es sich. Wenn Tн. Gomperz ${ }^{7}$ hier von dem «baconischen» Geist spricht, «der die ganze Schrift durchweht», so hat er mit diesem Vergleich zugleich ihre Atmosphäre und geistige Verwandtschaft aufs beste bestimmt.

Der Verfasser von de arte, der in der eben skizzierten Weise eine so eindrucksvolle Epideixis ärztlicher Methoden zu geben versteht, ist kein Arzt $^{8}$. Dies mag auch mit ein Grund sein, daß er mit so viel Zuversicht behauptet (de arte c.11.17,5 f. = G.53) : «Wenn aber die Natur die Erkenntnis gestattet, so wird sie auch die Heilung gestatten.»

Weitaus skeptischer in der Gesamthaltung wie in der Beurteilung der

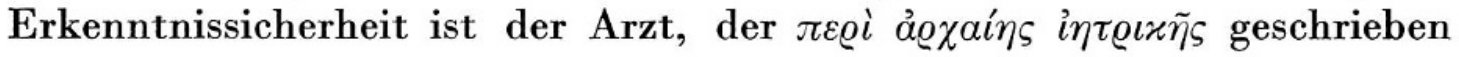
hat. Auch er verteidigt die Heilkunde und tut dies mit aller Energie, wobei er die aus Beobachtung und Erfahrung gewonnenen Behandlungsweisen jenen luftigen Schöpfungen entgegenstellt, die die Hypothesenmediziner ${ }^{9}$

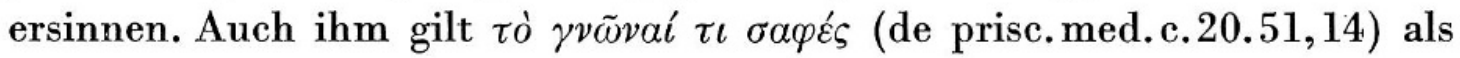
Idealforderung. Doch ist er sich auch der Grenzen seiner Wissenschaft und ihrer Methoden bewußt ${ }^{10}$. Mit Bedauern stellt er im 9. Kap. (41,19ff.) fest, daß die Heilkunst «nicht mit Zahl und Gewicht arbeite, sondern als Maßstab für ihre Diätvorschriften nur die Körperempfindung habe, so daß

${ }^{6} \mathrm{Da}$ diese Erkenntnisfindung nach den entwickelten Denkmethoden unserer Zeit in Wahrheit ein höchst komplexer Vorgang ist, bei dem das vierfache Methodengefüge von Analyse, Synthese, Induktion und Deduktion ineinandergreift, hat M. Hartmann in seinem Buche Die philosophischen Grundlagen der Naturwissenschaften, Jena 1948, S. $121 \mathrm{ff}$., dargestellt.

${ }^{7}$ Tн. GoMperz, a. O. 12 ; vgl. auch 140.

${ }^{8}$ Th. GomperZ, a. O. 2; W. Nestle a. O. 303.

${ }^{9}$ K. Deichgräber, Die Stellung des griechischen Arztes zur Natur, Die Antike 15 (1939), 129 ; W. NestLe, a. O. $236 \mathrm{ff}$.

${ }^{10}$ M. Pohlenz, Herodot. Neue Wege zur Antike, 2. Reihe, Heft 7/8, Berlin 1937, S. 198; A. J. Festugière, a. O. 41.44; W. Müri, Der Maßgedanke bei den griechischen Ärzten, Gymnasium 57 (1950), Heft 3, S. 183-201. 
Fehler unvermeidlich seien ${ }^{11} . »$ Diese Ungenauigkeit in den Methoden seiner so sehr geliebten Heilkunst ist dem Verfasser eine so schmerzliche Erkenntnis, daß er im 12. Kap. (43,27ff.) nochmals darauf zu sprechen kommt: «Aber schwer ist es, objektive Exaktheit zu erreichen, wo es so auf Genauigkeit in der Heilkunst ankommt ${ }^{12}$... Deshalb, so behaupte ich, darf man aber die Heilkunde nicht verächtlich abtun, als ob sie nicht bestünde und ihre Forschungen nicht richtig anstellte, wenn sie nicht in allen Dingen Genauigkeit besitzt, sondern ich bin vielmehr der Meinung: weil sie imstande war, durch vernunftgemäßes Überlegen von tiefer Unwissenheit zu einer fast vollkommenen Genauigkeit zu gelangen, müsse man ihre Entdeckungen bewundern ...»

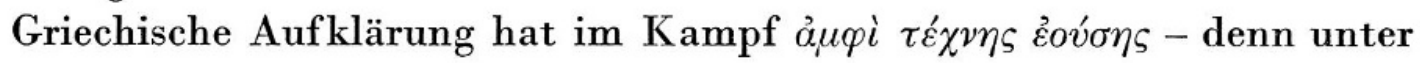
diese Formulierung hat die Zeit die verhandelten Probleme gestellt (de prisc. med.c. 1.36,7) - zweierlei Dinge ans Licht gehoben und gesichert: 1 . daß es eine Heilkunst gibt und diese über wohlgegründete Methoden verfügt, 2. daß aber diese Methoden es vielfach nicht gestatten, jenen Grad von Zuverlässigkeit oder Gewißheit zu erreichen, den die Kunst als wünschenswert erachten muß (de flat. 8, 8f.; de mul. VIII 94, 7L.).

Alle weitere medizinische Forschung seit der Antike steht im Zeichen des Ringens um diese Gewißheit. Alle versuchten und mißglückten Wege zu ihr aufzudecken, hieße eine Geschichte der Medizin unter einem vieles erhellenden Gesichtspunkt schreiben.

Es ist kein Zufall, sondern ein Zeugnis mehr für die innere Verwandtschaft der griechischen Aufklärung mit jener, die ganz Europa im 18. Jahrhundert bewegte, daß sich gerade in dieser Epoche die alten Fragestellun-

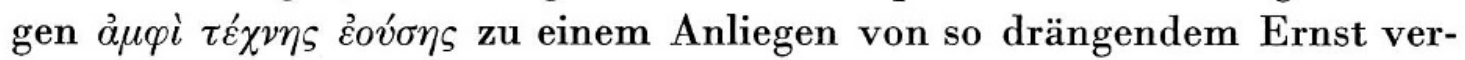
dichteten, daß eine Neuordnung der alten Argumente und Überprüfung des eigenen Standortes geboten erschien. Dies um so mehr, als selbst angesehene Ärzte ${ }^{13}$ des vorrevolutionären Frankreichs sich zu den Sottisen MoLIÈres ${ }^{14}$

11 M. Pohlenz, a. O. 198.

12 M. Pohlenz, a. O. 198, Anm. 1.

13 P.J.G. Cabanis, Du degré de certitude de la médecine, Didot-Paris 1798 (im folgenden zitiert als C. mit Seitenzahl), S. 118; J.P.FRANK, System einer vollständigen medicinischen Polizey, Bd. 6A. 1. Teil, Wien 1817, S. 63.

${ }^{14}$ Es ist die Pariser Fakultät des 17. Jahrhunderts, die im Tartuffe, Le malade imaginaire, L'amour médecin, Le médecin malgré lui karikiert wird. Dazu vgl. O. TeмкıN, Studien zum «Sinn»-Begriff in der Medizin, Kyklos 2 (1929), 66-84. 
bekannten und wie selbstverständlich die Angriffe eines J.J.Rousseau ${ }^{15}$ auf die Medizin mitmachten, die von dem viel gelesenen Emile aus weiteste Wirkung ausübten. In diesem wird bekanntlich die Heilkunst als schädlich hingestellt und der Einwand, daß ihre Fehler ärztlichem Versagen zuzurechnen seien, mit dem berühmt gewordenen, alle Gegner der Medizin einigenden Ausruf abgetan: «Gut, aber dann soll sie nicht mit dem Arzte zugleich kommen!» ${ }^{16}$ In dieser Atmosphäre, in der es in Paris zum guten Ton gehörte, se moquer de la médecine (C.118), erwuchs der Heilkunde ein neuer Verteidiger in dem französischen Arztphilosophen Pierre Jean Georges Cabanis (1757-1808) ${ }^{17}$. Indem er für seine im Revolutionsjahr 1789 verfaßte, aber erst 1798 erschienene Schrift den Titel $D u$ degré de certitude de la médecine wählte, machte er die innere Krise, in die die Heilkunde seines Jahrhunderts geraten war, weithin sichtbar.

Denn um eine innere Krise der europäischen Heilkunde handelt es sich am Ende eines Jahrhunderts, das wie kein anderes medizinische Systeme emporwachsen und wieder niedergehen sah. Diese hatten die Namen der groBen Kliniker der ersten Jahrhunderthälfte getragen, wie den eines BoERhaAve, Friedrich Hoffmann und Stahl, und sich schon dadurch empfohlen. Sie hatten aber ebenso wenig in der Praxis standhalten können wie um die Jahrhundertmitte die vitalistischen Ausformungen der Stahlschen Lehre durch Bordeu und Barthez in der Schule von Montpellier. Andere Versuche, ärztliches Denken und Handeln unter ein einheitliches Erklärungsprinzip zu stellen, waren von der altberühmten Schule von Edinburgh durch Cullen und Brown erfolgt, um dessen Lehre gerade im letzten Jahrzehnt des 18. Jahrhunderts in Deutschland heftige Kämpfe tobten. Sie waren noch nicht zu Ende gekämpft, die Willkürlichkeit auch dieser Lehren noch nicht voll eingesehen, als wieder neue emporschossen, anders nur im Inhalt, gleich aber immer in dem Anspruche, untrüglich und wahr zu sein: wir meinen jene, die von der naturphilosophischen Schule und von Hahnemann ausgingen. Daneben behandelte man noch weiter nach MesMER und diskutierte den Überfluß oder Mangel des Sauerstoffs als Ursache der Krankheiten oder die Infarzierung der Eingeweide und noch anderes mehr. «Die Unsicherheit der wissenschaftlichen und praktischen Medizin

${ }^{15}$ J.-J.Rousseau, Emil oder über die Erziehung, Übersetzt von E. von SallwürK, Langensalza 1882, 1. Band, 1. Buch, §95-105, S. $36 \mathrm{ff}$.

${ }^{16}$ J.- J. RousseaU, a. O. §98, S. 37.

${ }^{17}$ Reiche Literaturangaben zu Cabanis und dem Ideologenkreis in den unten angeführten Arbeiten von 0. Temkin, G. Rosen und E. H. ACKerKNecht. 
an der Schwelle des 19. Jahrhunderts», wie P. Diepgen ${ }^{18}$ diese Situation kennzeichnet, machte die besten Köpfe unter den Ärzten wirr. Wo war in dem unaufhörlichen Wechsel von Systemen, Schulen und Lehren das Bleibende und Sichere, an das man sich in der Praxis halten konnte? Wo war die Gewißheit in der Heilkunde? Gab es diese überhaupt und in welcher Form?

In diese innere Not europäischen Arzttums stieß nun Cabanis' Schrift vor und legte sie an ihrer Wurzel bloß. Der Verteidiger der Heilkunst wurde zugleich ihr scharfsichtiger Kritiker, indem er zunächst in sieben Einwürfen den Finger auf die brennenden Wunden seiner Wissenschaft legte, um dann von seinem Standort aus zu zeigen, wie man sie lindern bzw. heilen könne. Doch bevor wir ihm dabei folgen, haben wir zu fragen: Welcher Standort war denn der seine?

Wir dürfen uns hier kurz fassen und können dies tun dank drei vorzüglichen Arbeiten, die in den letzten Jahren Cabanis' geistige Heimat, die Struktur seines Denkens und seinen Einfluß auf die Pariser klinische Schule und auf die amerikanische Medizin des frühen 19. Jahrhunderts dargelegt haben. Schon dieser Hinweis auf den Inhalt der Arbeiten von G. Rosen ${ }^{19}$, O. Temkin ${ }^{20}$ und E. H. Ackerknecht ${ }^{21}$ läßt erkennen, daß wir es bei Cabanis mit einer Persönlichkeit von eminenter Wirkkraft zu tun haben. Sein geistiger Ahne ist Condillac. Mit ihm teilt er die Überzeugung, daß alle Erkenntnis auf Sinneswahrnehmung zurückgeht und alle Erscheinungen des Seelenlebens Komplexe von Empfindungen darstellen, die in ihre einzelnen Bestandteile zu zerlegen Aufgabe der Analyse ist. Also Aufgabe jener Methoden, von denen Cabanis 1789 in seiner Schrift (C. 4) mit Zuversicht behauptet: destinées à changer entièrement dans peu, la face du monde intellectuel. In dieser Überzeugung wird er mit DestutT de Tracy in jenem Kreis der Ideologen führend, die aus genauer Beobachtung von Tatsachen nur die sichersten Schlüsse ziehen, niemals reinen Hypothesen den Wert von Tatsachen zuweisen und das Eingeständnis absoluten Nichtwissens gegenüber jeder Behauptung vorziehen, welche sich nur auf Wahrschein-

18 P. Diepgen, Geschichte der Medizin, Berlin 1951, II/1, 44.

${ }^{19}$ G. Rosen, The Philosophy of Ideology and the Emergence of Modern Medicine in France, Bull. Hist. Med. 20 (1946), 328-339.

${ }^{20}$ O. Temкin, The Philosophical Background of Magendies Physiology, Bull. Hist. Med. 20 (1946), 10-35.

${ }^{21}$ E. H. Ackerknecht, Elisha Bartlett and the Philosophy of the Paris Clinical School, Bull. Hist. Med. 24 (1950), 43-60. 
lichkeit gründet ${ }^{22}$. Aus dieser Überzeugung heraus ist die Schrift $D u$ degré de certitude de la médecine geschrieben, an deren Anfang Cabanis die programmatische Erklärung setzt (C. 15): et c'est en partant de ces données positives, que je me propose d'examiner si par l'observation, et par les raisonnements simples qui s'en deduisent immédiatement, on peut donner une base solide aux principes de la médecine ...

Es ist also der Standpunkt eines empirischen Skeptizismus, von dem aus Cabanis die Krise der zeitgenössischen Medizin beurteilt und in sieben Einwürfen formuliert und diskutiert. Von diesen dürfen die ersten vier, die wir im folgenden eingehender behandeln, unser besonderes Interesse beanspruchen, während die übrigen drei (Fraglichkeit medizinischer Erfahrungen; Verschiedenheit bzw. Gegensätzlichkeit medizinischer Lehrsysteme; Schwierigkeit, ein vollkommener Arzt zu werden) notwendig aus den vorhergehenden erfließen.

Schon der erste Einwurf ist dazu angetan, die Grundfesten der Medizin zu erschüttern. Er beruht auf folgendem Räsonnement (C. 50 f.): Die Medizin beschäftigt sich mit der Erhaltung des Lebens, ohne das Lebensprinzip und die Kräfte zu kennen, die auf den Organismus wirken. Wie kann eine Kunst Haltbarkeit und Gewißheit haben, deren Grundlage unbekannt ist?

Dem stellt Cabanis die Frage entgegen: Zugegeben, wir kennen die Ursache des Lebens nicht, aber ist diese Kenntnis für uns denn auch notwendig? Wir kennen nicht jene letzte und wahre Ursache der Dinge, la force spontanée (C. 52), die sich den verzweifelten Bemühungen der Denker aller Zeiten entzieht. Wir können aber in den Phänomenen der Gesundheit und Krankheit die Äußerungen und Wirkungen dieser cause première beobachten; wir können weiters zwischen den Wirkungen und zwischen uns bestimmte Beziehungen feststellen, daraus unsere Schlüsse ziehen, sie durch wiederholte Beobachtungen bestätigen und davon praktische Regeln ableiten (C. 51.54). So kennen wir beispielsweise die Ursache der Verdauung nicht (C. 54f.), können aber - und hier schaltet Cabanis (C. $57 \mathrm{f}$.) die uns wohlbekannte Argumentation aus dem 5. Kap. von de arte (s. S. 153) ein beobachten, was nützlich und schädlich ist, und daraus bestimmte diätetische ${ }^{23}$ Regeln ableiten. Und das muß uns genügen.

${ }^{22}$ G. Rosen, a. O. 331.

${ }^{23}$ Hier, S. 59 f., schaltet Cabanis eine Erörterung über das Verhältnis von Diätetik und Arzneiwissenschaft ein, mit der er der bei CELsus wiedergegebenen Auffassung der Empirikerschule folgt. Vgl. K. DeICHGRÄBER, Die griechische Empirikerschule, Berlin 1930, S. 93, $10 \mathrm{ff}$. (im folgenden zitiert als D. mit Seite und Zeile). 
Im übrigen betrifft dieser erste Einwand nicht nur die Medizin, stellt Cabanis (C. $60 \mathrm{f}$.) fest, sondern gilt in gleichem Maße auch für alle übrigen Wissenschaften, die auf Beobachtungen und Erfahrungen beruhen. Die Heilkunde kann daher mit Recht fordern, alle die Grade von Gewißheit auch für sich in Anspruch nehmen zu dürfen, die diesen anderen empirischen Wissenschaften zukommen.

Gilt der erste Einwand nicht der Medizin allein, so trifft der zweite sie an ihrer Wurzel, indem er das ganze Gebäude der Heilkunde in Einsturz zu bringen droht. Er lautet: die Ärzte behaupten, eine Krankheit nur dann rationell heilen zu können, wenn sie ihre Ursachen beseitigen. Die Natur der Krankheiten und ihre nächsten Ursachen sind ihnen aber unbekannt (C.61).

Bevor wir auf die Entgegnung Cabanis' eingehen, möchten wir eine Stelle bei einem guten Praktiker des Jahrhunderts, den auch Friedrich II. an sein Krankenbett rief, bei Johann Georg Zimmermann ${ }^{24}$, zitieren, um an ihr zu illustrieren, wie eine solche «ursächliche» Behandlung in der Praxis aussah : «Man nimmt zum größten Nachtheil des Kranken die ersten Begriffe einer Krankheit aus ihrem innern und unsichtbaren Wesen. Zum Eckel wird von gesalzenem, dickem und verdorbenem Blute gesprochen, ohne daß man den geringsten Beweis hierüber führen könne. Nach diesen willkürlichen Säzen werden nichts destoweniger alle Erscheinungen einer Krankheit von den Practicis beurtheilt, die Anzeigen zur Heilung errichtet, die Methoden bestimmt, und die Arzneyen gegeben. Alle Ärzte, die mit der Kunst, die Krankheiten zu beobachten, unbekannt gewesen, haben in allen Zeiten ihre ganze Kenntnis der Krankheiten auf dieses elende Geschwäz gebaut. Ihre Namen und selbst ihre Definitionen flossen niemals aus dem äußern Wesen der Krankheiten, weil sie zu stolz das Sichtbare zu bemerken, allein auf das Unsichtbare bauten.»

Welche Ursachen sind denn gemeint, so fragt Cabanis (C. 65) in seiner Entgegnung. Jene, die die Alten verborgene (cachées) nennen oder die offenbaren (évidentes) ? Die letzteren zeigen sich durch sich selbst oder in ihren Wirkungen, sind also beobachtbar, mithin ist die Krankheit erkennbar und kann behandelt werden. Die ersteren können dem Arzte gleichgültig sein; je mehr sie sich seinem Fassungsvermögen entziehen, desto entbehrlicher sind sie ihm.

Wenn wir nun zu dieser Stelle jene hinzusetzen, die uns bei Celsus (92, 1 ff. D.) über die Lehre der Empiriker überliefert ist, so wird mit einem

${ }^{24}$ J.G.ZimmermanN, Von der Erfahrung in der Arzneykunst, Zürich 1763, 1. Teil, S. 251. 
Schlage der Quellbezirk sichtbar, aus dem Cabanis seine agnostische Haltung in der Ursachenfrage herleitet: Ii qui se empiricos ab experientia nominant, evidentes quidem causas ut necessarias amplectuntur: obscurarum vero causarum et naturalium actionum quaestionem ideo supervacuam esse contendunt, quoniam non comprehensibilis natura sit.

Cabanis selbst weist uns auf diese Abhängigkeit hin, indem er eine alte Streitfrage zwischen Dogmatikern und Empirikern (99, $22 \mathrm{ff}$.; 307 D.) zur Erläuterung der von ihm und den Empirikern vertretenen Auffassung heranzieht (C. 63 f.): Ein Mann wird von einem tollwütigen Hunde gebissen. Die Dogmatiker fragen nun die Empiriker, um sie ihrer absurden Haltung in der Ursachenfrage zu überführen, ob es ihnen gleichgültig sei, ob dieser $B i ß$ von einem tollwütigen Hunde stamme oder nicht. Durchaus nicht, antworten die Empiriker; aber daß der Biß von einem tollwütigen Hunde stammt, betrachten wir nicht als Ursache, sondern als bloßes Faktum, welches zur Geschichte der Krankheit gehört. Hier geht es letztlich um Worte, stellt Cabanis fest, bekennt aber doch eindeutig, daß seine Sympathie der empirischen Auslegung gilt (C. 65).

Aus dieser anätiologischen Haltung der Empiriker ergibt sich notwendig ein anderes Charakteristikum ihrer Lehre, das Cabanis ebenfalls von ihnen übernommen hat: die Abwertung der ursächlichen Krankheitsbenennung zugunsten der semeiotischen (vgl. 309 D.) ${ }^{25}$. Was ist denn der Seitenstich, la pleurésie, fragt Cabanis (C. 61) ? Doch nichts anderes als le concours de ces accidents qui la constitue, nämlich des Hustens, des Blutauswerfens, des Seitenschmerzes, der Dyspnoe usw. Das Wort «pleurésie» dient nur dazu, uns die Gegenwart all dieser charakteristischen Symptome in Erinnerung zu rufen. Krankheit ist also ein Symptomenkomplex. Das aber ist ein Hauptdogma der antiken Empirikerschule (309 D.). Wenn also Cabanis die Krankheit als le concours des accidents definiert, so ist diese Definition nichts anderes als die Übersetzung des griechischen $\sigma v v \delta \varrho \mu \eta \dot{~}$

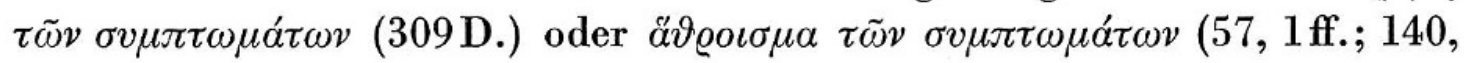
$11 \mathrm{ff}$.$) .$

Wir möchten hier noch auf ein «Grundbekenntnis der empirischen

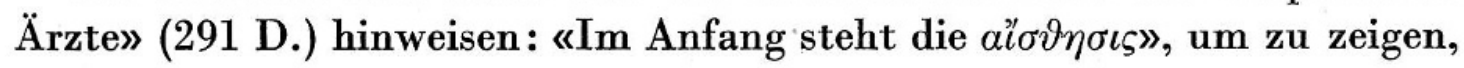

${ }^{25}$ Die gleiche Auffassung finden wir auch bei J.G.ZimmermanN, a. O. 252: «Die unmittelbar von den vermeinten nächsten Ursachen der Krankheit hergenommenen Namen geben uns nichts als falsche Begriffe.» Zimmermanns eingehende und warme Würdigung der antiken Empirikerschule a. O. 49 ff. sowie die Stelle S. 245 lassen keinen Zweifel, daß auch er hierin direkt von den Empirikern abhängig ist. 
wie auch ihrerseits die antike Empirikerschule durch ihre erkenntnistheoretische Haltung den sensualistischen Lehren entgegenkam, die über CoNDILlac Cabanis' philosophisches Glaubensbekenntnis wurden ${ }^{26}$. Dieser gemeinsame erkenntnistheoretische Ausgangspunkt mag es Cabanis leicht gemacht haben, sich auch die übrigen Hauptpunkte der antiken Empirikerlehre so zu eigen zu machen, daß wir von einer medizinischen Renaissance des Empirismus am Ausgange des 18. Jahrhunderts durch Cabanis sprechen können.

Was bedeutet nun diese Renaissance des Empirismus für die früher skizzierte Situation, in der sich die europäische Heilkunde befand ? Sie bedeutet dasselbe, was die antike Empirikerschule für ihre Zeit gewesen war: Abkehr von einer dogmatischen Medizin mit ihren supponierten Ätiologien und voreiligen Systembildungen und Hinwendung und Beschränkung auf eine nüchterne Tatsachenforschung, die sich mit der Beobachtung der Phänomene und gewisser daraus abgeleiteter Regeln begnügt. Bei dem damals noch unentwickelten Stand der pathologischen Anatomie, bei dem gänzlichen Fehlen jener Einsichten, die später die Bakteriologie erschloß, war es sicherlich nicht die schlechteste Auskunft, sich auf die Erklärung zu beschränken: Krankheit ist ein Symptomenkomplex.

Der dritte Einwurf gegen die Unzuverlässigkeit der Heilkunde fußt auf der Unsicherheit ihrer Diagnostik (C. 101 ff.). Bei den zahlreichen Veränderungen, die die Krankheiten je nach Alter, Temperament und Lebensweise des Patienten, nach Jahreszeit, Klima und epidemischer Konstitution aufwiesen, sei es unmöglich, eine klare Scheidung und Zuweisung der einzelnen Krankheitszeichen an die betreffende Krankheitsform vorzunehmen. Man sei gezwungen, ständig Ausnahmen von den Regeln zu machen, so daß schließlich dem Praktikus am Krankenbette nichts anderes übrigbliebe, als sich auf seinen Instinkt zu verlassen.

Diese Kritik an der Diagnostik wird am Ende eines Jahrhunderts geübt, das wie kein anderes sich um die systematische Erfassung der Krankheiten in den Nosologien eines Sauvages, SAgar, Vogel und Cullen ${ }^{27}$ bemüht hatte und, wie P. Diepgen a. 0.31 feststellt, auch nicht wenig zur besseren

${ }^{26}$ Dieses findet er auch in der auf $\tau \varrho \iota \beta \eta$ und $\lambda o \gamma \iota \sigma \mu o ́ s$ beruhenden Erkenntnislehre der

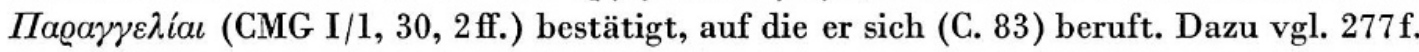

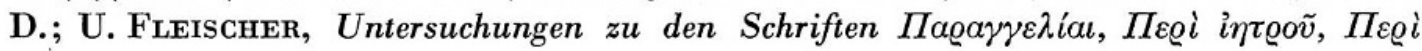

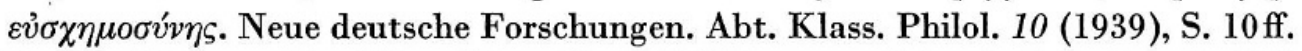

${ }^{27}$ PInels Nosographie philosophique (Paris 1793) war zur Zeit, als Cabanis seine Schrift verfaßte (1789), noch nicht erschienen. 
Beschreibung der Symptome beitrug. Die verwirrende Fülle von Zeichen machte eine Anordnung nach gewissen Gesichtspunkten notwendig. Um das dabei geübte Verfahren kennenzulernen, wollen wir wieder den uns schon bekannten Johann Georg Zimmermand ${ }^{28}$ verhören: «Die Kenntnis des wesentlichen und außerwesentlichen in denselben (sc. den Krankheiten) führt uns auf die Kenntnis ihrer Ähnlichkeiten und Unähnlichkeiten, die Kenntnis einzelner Zufälle auf die Kenntnis der zusammengesezten, die Kenntnis einfacher Krankheiten auf die Kenntnis der zusammengesezten. Aus dem Begriffe vieler einzelner Krankheiten entstehet allmählig der Begriff ihrer verschiedenen Abhänglichkeiten und ihrer Beziehungen auf derselben ganzes System.»

Auch Cabanis verkennt die Notwendigkeit einer solchen Ordnung nicht (C. 73), muß aber an den bisher erschienenen Nosologien die willkürliche und vorzeitige Form ihrer Klassifikation tadeln. Die Ordnung der Krankheiten, wie er sie meint, hat die Natur selbst entworfen, und die großen ärztlichen Beobachter aller Zeiten haben sie auch bereits beschrieben. Nach dieser Ordnung, so meint er, ist es möglich, die hervorstechendsten Merkmale der Krankheiten zu beobachten, regelmäßige Verbindungen mit gewissen vorhergehenden und gegenwärtigen Tatsachen festzustellen und daraus die Verkettung von Ursache und Wirkung zu bestimmen. Die Beobachtung kann ferner diese Kenntnis auf bestimmte Regeln zurückführen und in diesem Rahmen eine Diagnostik ermöglichen.

Cabanis sagt aber im Zuge dieser Erörterungen nichts über den Sicherheitsgrad aus, den die von ihm vorgeschlagene diagnostische Methode in der Praxis bieten konnte. Wenn wir uns überlegen, daß diese Methode vor allem auf einer generalisierenden Induktion beruht, die bei dem damaligen Wissensstand notwendigerweise nicht wirkliche, in unserem Sinne objektive Wesenszüge der Krankheiten erfaßte, sondern oft Scheinanalogien herstellte, dann können die Ergebnisse eines solchen Verfahrens dem damaligen Arzt keine große Sicherheit am Krankenbett geboten haben. Cabanis' Rezensent in der Salzburger medicinisch-chirurgischen Zeitung ${ }^{29}$ bestätigt uns dies, von dem Johann Stoll ${ }^{30}$, ein Verwandter des berühmten Wiener Klini-

${ }^{28}$ J.G.ZimmermanN, a. O. $245 \mathrm{f}$.

${ }^{29}$ Medicinisch-chirurgische Zeitung, herausgegeben von JoH. Jac. HartenkeIL, Salzburg, 1 (1799), Nr. 23, S. 408.

${ }^{30}$ J. SтоLL, Staatswissenschaftliche Untersuchungen und Erfahrungen über das Medicinalwesen nach seiner Verfassung, Gesetzgebung und Verwaltung, 2 Bände, Zürich 1812, 1. Band, S. 19. 
kers Maximilian Stoll, für eine so gut beobachtete Krankheitsform wie die Lungenentzündung folgende Äußerung übernimmt: «Jeder Arzt, der diese Krankheit oft behandelt hat, gebe der Wahrheit die Ehre und gestehe, wie oft er die Lungenentzündung, zum großen Nachtheile seiner Kranken, entweder bis zum Tode derselben verwechselt, oder erst dann, als er mit seinen Mitteln nichts mehr auszurichten vermochte, unterschieden habe ?» So kann diese und andere Erfahrungen präzisierend und zusammenfassend J. KöllneR ${ }^{31}$ über den Wert der Semeiotik am Jahrhundertende folgendes Urteil abgeben: «In vielen ihrer Sätze herrscht ja oft nur einmalige Erfahrung, in mehreren gesteigerte, und nur in sehr wenigen bis zum höchsten Grade gebrachte Wahrscheinlichkeit, die bis an die Gewißheit grenzet.»

Diese vielleicht nicht immer so klar bewußte, aber doch gefühlte Unsicherheit der Semeiotik läßt Cabanis über alle oben verhandelten zuversichtlichen Erörterungen seiner Methode hinweg an einer anderen Stelle seiner Schrift zu der Feststellung kommen (C. 117): En médecine, tout, ou presque tout dépendant du coup-d'œil et d'un heureux instinct, les certitudes se trouvent plutôt dans les sensations mêmes de l'artiste, que dans les principes de l'art (vgl. auch C.67).

Nehmen wir diese Feststellung im vollen Sinne ihrer Aussage, so drückt sie eine tiefe Resignation über die Möglichkeit aus, sich auf Grund bestimmter Prinzipien Gewißheit zu verschaffen. Indem Cabanis hier bei der Verwertung ärztlicher Beobachtung - und auch C. 67 - dem Instinkt, also einem irrationalen Faktor, zur Erlangung dieser Gewißheit einen Hauptanteil zuerkennt, so bezieht er mit diesem Regreß auf den Instinkt eben jenen Standpunkt, der damals zu so schweren Zweifeln am Wert der Medizin Anlaß gegeben hatte; ja es ist ferner festzustellen, daß er hier nicht mehr in vollem Einklang mit seinem eingangs verkündeten Programm steht, durch Beobachtungen und einfache Vernunftschlüsse den Grundsätzen der Medizin eine feste Basis zu geben (C. 15). Nun haben bereits die amerikanischen Arbeiten darauf hingewiesen ${ }^{32}$, daß Cabanis not a very concise thinker ist. Wir müssen allerdings die besonderen Einflüsse in Rechnung setzen, unter denen Cabanis gerade bei dieser Äußerung steht. Da ist einmal Rousseau, dessen hohe Bewertung des Instinktes

\footnotetext{
${ }^{31} \mathrm{~J}$. KöLLNER, Ist die Heilkunde als Wissenschaft betrachtet möglich und wie ist sie es? Magazin zur Vervollkommnung der theoretischen und praktischen Heilkunde, herausgegeben von A. Röschlaub, 1 (1799), $329 \mathrm{f}$.

32 O.Temkin, a. O. 20.
} 
bei Mensch und Tier einen gewaltigen Eindruck hinterlassen hatte ${ }^{33}$. In noch stärkerem Maße steht jedoch Cabanis unter dem Einflusse der eingangs bereits herangezogenen Schrift de prisc. med., die er selbst zitiert (C. 117). Dabei ist ihm ein Interpretationsfehler unterlaufen, der ihm die Übereinstimmung der griechischen Schrift mit sensualistischer Erkenntnistheorie im Sinne Condillacs besonders sinnfällig zu machen schien.

Es handelt sich um die bereits erwähnte Stelle des 9. Kap. (41, $21 \mathrm{ff}$.), in der es darum geht, in der Heilkunde einen Maßstab für die Diätvorschriften zu finden. Wir geben sie in der Übersetzung von K. DEICHGRÄBER ${ }^{34}$ : «Man muß einen bestimmten Maßstab ins Auge fassen. Man dürfte aber als Maßstab weder eine Zahl noch etwa ein Gewicht noch etwas anderes finden, an dem man das exakt Richtige ablesen könnte, außer $\tau o \tilde{v}$

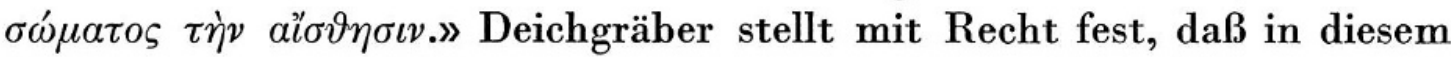
Zusammenhang $\tau o \tilde{v} \sigma \omega ́ \mu \alpha \tau o \varsigma$ nur Gen. possessivus, nicht objectivus sein kann, so daß sich nach W. MÜRI ${ }^{35}$ folgende Übersetzung ergibt: «der Empfindlichkeit des Körpers» (nämlich des Patienten). Cabanis hingegen hat aı $\sigma \vartheta \eta \sigma \iota \varsigma$ auf den beobachtenden Arzt und dessen Wahrnehmungen bezogen (C. 117) und derart in der griechischen Stelle die Grundlage für seine Behauptung gefunden: ... les certitudes se trouvent plutôt dans les sensations mêmes de l'artiste...

Der hervorragende Physiologe und Arzt Johann Christian Reil (1759-1813) stellt am Ende des Jahrhunderts in seiner Fieberlehre ${ }^{36}$ fest: «Unsere Erkenntniß der Wirkung der Arzneyen ist empirisch. Was wir von verändernden, blutreinigenden, die Säfte verbessernden, auflösenden, einschneidenden Mitteln sprechen, ist größtentheils nichts anders, als eine sinnlose Tradukzion aus der todten Natur in die lebendige. Bis jetzt sind uns von den wenigsten Mitteln ihre Bestandtheile bekannt, wenigstens fehlt es uns ganz an einer Erkenntniß des quantitativen Verhältnisses derselben, das doch die Wirkungen des Mittels so manchfaltig abändert. Wir

${ }^{33}$ Man vgl. in dieser Hinsicht auch Cabanis' Ausführung über die Entstehung der Heilkunde (C. $28 \mathrm{ff}$.), in der er in einer dem Verfasser von de prisc. med. analogen Weise seine eigene Methode in jene der primitiven Medizin hineinprojiziert und die instinktgemäße Behandlung als Vorstufe der Heilkunde ansieht (C. 34). Man vgl. auch die Erörterung über den Instinkt in den Schriften Magendies bei O. Temkin, a. O.15.

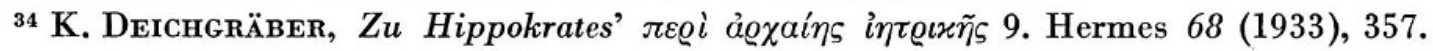

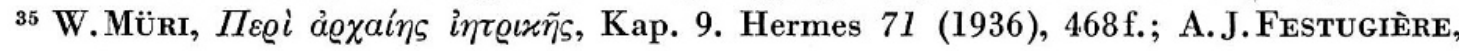
a. 0.7 .41 .

${ }^{36}$ Joh. Christ. ReIL, Über die Erkenntniß und Cur der Fieber, Halle 1799, § 15, S. 21. 
wissen nicht, auf welche Art die Arzneyen im Körper verändert, in ihre Bestandtheile zerlegt, und zu neuen Substanzen verbunden werden. Wir wissen nicht, was sie für Veränderungen in der Mischung und Form der thierischen Materie verursachen; wie sie dieses thun, auf welche Organe sie unmittelbar und auf welche sie mittelbar wirken. Der Zusammenhang der Veränderungen zwischen der Anwendung der Arzneyen und ihren letzten in die Sinne fallenden Erscheinungen ist uns gänzlich unbekannt.»

Was Reil hier mit Bedauern und einer so schönen Offenheit feststellt, ist gerade das, was als viertes Argument gegen die Zuverlässigkeit heilkundlicher Methoden bei Cabanis diskutiert wird (C. $17.82 \mathrm{ff}$.): daß nämlich Natur und Wirksamkeit der Heilmittel gänzlich unbekannt seien. Darüber haben sich die besten und verantwortungsbewußtesten Ärzte der Zeit keinem Zweifel hingegeben. Sie wußten zwar, daß man mit Quecksilber die Syphilis, mit China das Wechselfieber heilen, mit Kanthariden auf der Haut Blasen ziehen und mit einer Anzahl anderer Mittel andere Wirkungen hervorbringen könne; aber wie diese Wirkung im Organismus zustande käme, ob atomistisch, dynamisch oder sonstwie und auf Grund welcher Bestandteile, das entzog sich ihrer Kenntnis ${ }^{37}$. Und diese Kenntnis wurde um nichts bereichert, sondern es mußte nur noch zu größerer Unsicherheit und Verwirrung führen, wenn so berühmte Ärzte der Zeit, wie BoerhaAve, Friedrich Hoffmann, van Swieten, Linné, Darwin und Brown, die alle selbst an Podagra litten, die verschiedensten, ja einander direkt entgegengesetzten Heilverfahren bei der Behandlung dieser Krankheit als an sich selbst erprobt und untrüglich empfahlen ${ }^{38}$. «Der Apparatus medicaminum ist weiter nichts, als eine sorgfältige Sammlung aller Trugschlüsse, welche die Ärzte von jeher gemacht haben. Einige richtige Erfahrungsurteile sind darunter; wer mag aber seine Zeit darauf verwenden, diese wenige Goldkörner aus dem ungeheuren Misthaufen heraus zu suchen, den die Ärzte seit zweitausend Jahren zusammen geschleppt haben.» Dieses Urteil fällt Christoph Girtanner ${ }^{39} 1798$ in Deutschland, also im selben

${ }^{37}$ J. SToLL, Versuch einer medicinischen Beobachtungskunst, Zürich 1802, S. 152f.; id., Staatswissenschaftliche Untersuchungen ... $20 \mathrm{f}$; L. J.Schmidtmann, Ausführliche praktische Anleitung zur Gründung einer vollkommenen Medizinal-Verfassung und Polizey, Hannover 1804, 1. Band, $155 \mathrm{f}$.

${ }^{38}$ Georg von Wedekind, Über den Werth der Heilkunde, Darmstadt 1812, S. 144f.

${ }^{39}$ Christ. Girtanner, Ausführliche Darstellung des Brownischen Systemes der praktischen Heilkunde, Göttingen 1798, 2. Band. S. 600. G. v. WEDEKIND, a. O. 142; vgl. ein ähnliches Urteil bei J. StolL, Versuch ... 434. 
Jahre, als Cabanis' Schrift in Paris erschien. In seiner vielleicht allzu großen Härte offenbart es nur um so deutlicher die Krise der Therapie am Jahrhundertende. So erwuchs nicht zum letzten aus Mißtrauen gegen die Trugschlüsse der anderen die Gepflogenheit, daß fast jeder Praktikus seine eigene Materia medica hatte, einen beschränkten Schatz von Heilmitteln, die er selbst erprobt hatte und auf deren Anwendung er sich im gegebenen Falle verlassen zu können meinte ${ }^{40}$.

Cabanis (C.110f.) empfiehlt dieses Verfahren sehr warm, stellt es doch die Verwirklichung seiner für die Therapie empfohlenen Methode dar, die Wirkungen der Arzneikörper nach verschiedenen Richtungen hin auszuforschen, durch weitere Erfahrungen zu bestätigen und diese in Regeln festzuhalten (C. 82f.). Auf eine Erforschung der Natur und der Zusammensetzung dieser Arzneikörper jedoch glaubt Cabanis gänzlich verzichten zu können, da es für uns nicht von Nutzen sei zu wissen, quelle est la nature de quinquina, pour remarquer son pouvoir spécifique dans les fièvres intermittentes (C. 83). Dies stellt er in einem Skeptizismus fest, that goes far beyond what is usually called metaphysical agnosticism ${ }^{41}$.

Es ist kein Zweifel, daß die Krise ärztlichen Denkens - denn daß es sich um eine solche in weitestem Ausmaß am Ende des 18. Jahrhunderts handelt, wird klar geworden sein - in keinem Bereich der Medizin so offenkundig zutage liegt und so allgemein eingesehen ist wie in dem therapeutischen. Denn die empirische Reaktion Cabanis' ist ja zunächst mehr ein resigniertes Einbekenntnis dieser Krise, als daß sie sie durch positive Leistungen überwunden hätte. Es sollte erst der Zeit nach Cabanis vorbehalten sein, aus seiner "philosophy of observation» eine «medicine of observation» zu entwickeln und aufzubauen ${ }^{42}$.

Zwar will Cabanis seine durch Beobachtung und Erfahrung gewonnenen Regeln sur toutes les bases des certitudes humaines (C. 83) gegründet wissen. Wenn wir aber fragen, welche Gewißheit dies ist, dann sagt uns Cabanis selbst, daß es nicht jene sein kann, wie sie die Mathematik charakterisiert (C. 87), sondern, qu'il suffit de prouver rigoureusement la certitude de la médecine, telle que la nature bien interrogée l'enseigne aux hommes (C. 104). Hier enthüllt sich uns als Cabanis' Quelle der Gewißheit die Natur. Aber ist es jene Natur, wie er sie uns durch seine ganze Schrift gezeigt hat

${ }^{40}$ L. J.SChmidtmanN, a. O. 173; G. v. Wedekind, a. O.148.

${ }^{41}$ E. H. ACKERKNeChT, a. O. 52.

42 E. H. Ackerknecht, a. O. 50. 
als stete Lehrmeisterin des Menschen zufolge ihrer Regelmäßigkeit, ihrer Beständigkeit, ihrer Ordnung (C. 54.143)? Die Natur, aus der Cabanis das Abbild seiner Gewißheit herleitet, ist eine andere; sie ist eine, die in nichts l'exacte précision bringt; elle semble avoir voulu se conserver par-tout une certaine latitude, afin de laisser aux mouvements qu'elle imprime, cette liberté régulière qui ne leur permet jamais de sortir de l'ordre, mais qui les rend plus variés, et leur donne plus de grâce (C. 116). Es ist also eine Natur, die sich in der Mannigfaltigkeit, Abwechslung und Grazie gefällt und sich darin einen bestimmten Spielraum läßt, es ist eine Natur, die «sich der exakten Berechnung und eindeutigen Erklärung leicht entzieht, also zwar nicht absolut unfaßbar, aber aus anderen als rationalen und rationellen Prinzipien herzuleiten ist ${ }^{43} . »$ Es ist die natura varie ludens, die $\varphi v \sigma \iota \varsigma$

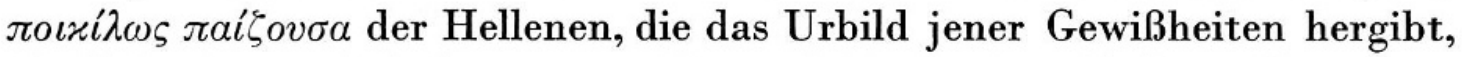
die Cabanis «praktische» nennt. Denn gleich im Anschluß an die zuletzt zitierte Stelle (C. 116f.) fährt Cabanis fort: Les vraies certitudes, en prenant ce mot dans son acception la plus stricte, appartiennent exclusivement aux objects de pure spéculation: dans la pratique, il faut se contenter d'approximations plus ou moins exactes, que par cette raison on pourroit appeler certitudes pratiques. Il faut s'en contenter, parceque ce sont les seules auxquelles la nature nous permette d'arriver, et parcequ'elles suffisent à l'espèce humaine pour assurer sa conservation et son bien-être.

Wir haben gesehen, daß in allen entscheidenden Punkten seines Werkes und seiner Lehre Cabanis aus antikem Gedankengut schöpfte ${ }^{44}$, es verlebendigte und in die Diskussion seiner Zeit hob. Auch dort, wo er von dem Spielraum des Unbestimmbaren in der Natur spricht und aus ihm seinen Hauptbegriff der certitudes pratiques entwickelt, steht er in der Tradition antiken Denkens. Es ist dies jene nachmals soviel diskutierte Konzeption, mit der der französische Arztdenker in seinem Ringen um die Grundlagen der Medizin Resignation und Zuversicht gleichzeitig bekundet hat.

E.H.Ackerkneght, G. Rosen und O.Temkin ${ }^{45}$ haben in ihren Arbeiten dargelegt, welcher Strom von Wirkung von dem ärztlichen Denker der Re-

${ }^{43}$ K. DeichgräBer, Natura varie ludens, Abh. geist. soz. wiss. Kl. Akad. Wiss. Lit. Mainz 1954, Nr. 3, S. 68. Dort das antike Stellenmaterial sowie Hinweise auf die Verbreitung dieser Naturvorstellung im 18. Jahrhundert.

${ }^{44}$ Für de arte hat bereits Th. GompERz, a. O. 13. 160, darauf hingewiesen.

${ }^{45}$ Zitiert unter Anmerkung 19, 20, 21. 
volutionszeit zur Pariser klinischen Schule hinüberging. Sie haben im einzelnen gezeigt, wie in Pinel, Bichat, Lä̈nnec, Louis, Magendie, RiCHERAND, um nur einige von den Großen dieser Epoche zu nennen, die ideologische Lehre Cabanis' weiterwirkte und vor allem durch die Anwendung der von ihm empfohlenen analytischen Methode die fruchtbarsten Ergebnisse zeitigte. Im besonderen ist E.H.Ackerknecht den Einflüssen Cabanis' und der Pariser Schule nachgegangen, die über Elisha Bartlett eine neue Ära der amerikanischen Medizin einleiteten.

So gut wie gar nicht hat man sich bislang um den Einfluß gekümmert, den Cabanis auf das ärztliche Denken in Deutschland ausübte. Und doch ist auch er sehr stark gewesen, hat Wirkungen der verschiedensten Art ausgelöst und weist eine besondere Note auf, von der im folgenden die Rede sein soll.

Schon die ausführliche und rasche Besprechung, die der Rezensent der Salzburger medicinisch-chirurgischen Zeitung ${ }^{46}$, eines damals im deutschsprachigen Gebiet sehr angesehenen kritischen Organs, der Schrift Cabanis' widmete, beweist das große Interesse, das man ihr jenseits des Rheins entgegenbrachte. Die Besprechung erschien bereits am 21. März 1799 und erstreckt sich auf zwei Nummern der genannten Zeitschrift. Zwar befürwortet der Rezensent auf das wärmste den Zweck der Schrift, nämlich den Glauben an die Heilkunde wiederherzustellen; aber um nichts weniger tadelt er den hiezu von Cabanis eingeschlagenen Weg. Er sei der einer unzureichenden rationellen Empirie, mit der man wohl Regeln, niemals aber apriorische allgemeingültige Gesetze in der Medizin erlangen könne. Letztere seien aber in einer Wissenschaft, «worin bey jedem Irrthume Menschenleben auf dem Spiele stehen», notwendig.

Diese Haltung ist für eine Richtung der deutschen Medizin am Jahrhundertende kennzeichnend. Wir meinen jene, die sich unter dem Einfluß der Kantschen Philosophie bemüht, die Medizin als systematische Wissenschaft zu erweisen, die unter leitenden Ideen steht, und die sich dabei in die Netze des Brownianismus verstrickt. Diese Richtung, der der Rezensent der Salzb. med.-chir. Zeitung sichtlich angehört, muß notwendig in scharfen Widerspruch zu dem früher entwickelten Grunddogma Cabanis' kommen, daß wir die Ursache von Leben und Krankheit nicht erkennen können. Wir werden dieser durch KANT und BRown bestimmten Richtung alsbald wieder begegnen, zunächst aber haben wir einige Reaktionen auf Cabanis zu verzeichnen, bei denen diese Faktoren im Hintergrund bleiben.

${ }^{46}$ Medic.-chir. Zeitung 1 (1799), Nr. 23, 401-409; Nr. 24, 417-422. 
Daß bereits 1799 eine deutsche Übersetzung von Cabanis' Schrift erschien, hat die Auseinandersetzung mit ihr wesentlich erleichtert. Sie stammt von dem Göttinger Arzt August Friedrich Ayrer ${ }^{47}$, der während seines sechsjährigen Pariser Aufenthaltes mit den neuen Strömungen in der französischen Medizin, insbesondere mit den Gedanken Cabanis', bekanntgeworden war und diese, wie aus der Einleitung und noch mehr aus den Anmerkungen hervorgeht, ganz zu den seinen gemacht hatte.

Noch im selben Jahre 1799 erschien eine Königsberger Dissertation, die von dem Sohn des dortigen Medizin-Professors Chr. Fr. Elsner, Christoph Joh. Heinr. Elsner ${ }^{48}$, stammt. Sie verficht den empirischen Standpunkt Cabanis', will Hypothesen aus der Praxis verbannt und an Stelle dessen die certitudes pratiques als Grundlage der Krankenbehandlung eingeführt wissen.

Aber diese Reaktionen auf Cabanis' Schrift, so aufschlußreich sie im einzelnen für die Haltung und Lage der deutschen Heilkunde sein mögen, treten doch an Bedeutung zurück vor der Auseinandersetzung, die mit dem französischen Arztdenker in einem anderen Bereich der Medizin stattfand. Wir meinen jenen, den eben Johann Peter Frank erschlossen hatte, indem er erstmalig das ganze Material der Hygiene in seinem System einer vollständigen medicinischen Polizey ${ }^{49}$ zusammenfaßte und darin eindringlich die Bedeutung einer rationellen Gesundheitspolitik für das allgemeine Staatswohl demonstrierte. Diese eben geschaffene Hygiene oder Staatsarzneikunde, wie sie sich damals noch in Verbindung mit der Gerichtsmedizin nannte, stand im Mittelpunkt des Interesses der Zeit. Die besten Köpfe unter den Ärzten, wie Franz Anton $\mathbf{M A I}^{50}$, wandten sich ihr zu. Durchdrungen von der großen Wohlfahrtsidee der Aufklärung wollten sie auch ihrerseits durch Entwürfe und Vorschläge über Medizinalverfassungen einen Beitrag zur physischen Glückseligkeit der Staatsbürger leisten. In keiner Zeit schossen mehr Vorschläge über medizinische Gesetzgebung, Medizinalverwaltung und Verfassung aus dem Boden, als gerade um 1800

${ }^{47}$ Aug. Friedr. Ayrer, Über den möglichen Grad der Gewißheit in der Arzneiwissenschaft, Göttingen 1799. Dazu vgl. die Besprechung in der Salzb. medic.-chir. Zeitung 3 (1799), $28-30$.

${ }^{48}$ Christoph. Ionnn. Henr. Elsner, Diss. inaug. de incerti in arte medica fonte, d. 3. Oct. 1799, Regiomont.

${ }^{49}$ J.P. Frank, System einer vollständigen medicinischen Polizey, Mannheim $1779 \mathrm{ff}$.

${ }^{50}$ Über ihn vgl. A. Fischer, Geschichte des deutschen Gesundheitswesens, Berlin 1933, 2. Band, S. $4.7 \mathrm{ff}$. 
und in den Jahren unmittelbar darnach. Außer dem hohen Interesse, das man damals dem neu erschlossenen Fach entgegenbrachte, hatte dies auch seinen Grund in den äußeren politischen Verhältnissen. Diese machten, durch die Koalitionskriege in Deutschland von Grund auf geändert, seit dem Reichsdeputations-Hauptschluß vom 25. Februar im Jahre $1803^{51}$ eine Reorganisierung fast aller Verwaltungszweige notwendig. So bot sich den Ärzten gerade in diesem Augenblick die Gelegenheit dar, die Errungenschaften des neu kreierten Faches in einer neuen bzw. verbesserten Medizinalgesetzgebung zu verwirklichen.

Diese Versuche erfolgten also gerade in dem Augenblicke, in welchem Cabanis' Schrift, wie früher gezeigt, ihre Wirkung in Deutschland zu entfalten begann und die Grundlagen der Medizin auf breitester Basis zur Diskussion stellte. Zu den dabei aufgerührten Fragen mußte jeder medizinische Gesetzgeber Stellung nehmen, um sich in ernster Auseinandersetzung mit ihnen über Sinnhaftigkeit oder Unzulässigkeit seines Vorhabens Klarheit zu verschaffen.

Einer solchen Auseinandersetzung begegnen wir gleich 1804 in LUDwIG Joseph Schmidtmanns (1764-1840), eines praktischen Arztes zu Melle im Osnabrückischen, Ausführlicher praktischen Anleitung zur Gründung einer vollkommenen Medizinal-Verfassung und Polizey ${ }^{52}$, die der bekannte LeBrecht Friedrich Benjamin Lentin mit einer Vorrede ausgezeichnet hat. Hier handelt das 2. Kapitel des 1. Bandes, das nicht weniger als 131 Seiten umfaßt «Von dem hohen Werthe der empirisch-rationalen Heilkunde und ihren großen Verdiensten um das Lebensglück der Menschen. Bestreitung der ihr gemachten Vorwürfe.» Es stellt nichts anderes als einen Auszug aus der Schrift Cabanis' dar, der der Autor in Argumentation und Gegenargumentation Punkt für Punkt folgt. Auf S. 177 nennt Schmidtmann ausdrücklich Cabanis als seine Quelle. Da Schmidtmann aus der Schule des von Cabanis so sehr geschätzten Wiener Klinikers Maximilian Stoll (C. 70) kommt, so mag es ihm nicht schwer gefallen sein, Cabanis' Gedankengänge so gut wie zur Gänze zu übernehmen. Allerdings bleiben dabei die skeptischen Vorbehalte Cabanis' mehr im Hintergrund, während die positive Argumentation schärfer betont, in den Vordergrund gerückt und durch neue Beweise verstärkt wird. Auf dieser Basis gelangt Schmidtmann (S.

${ }^{51}$ J.StoLl, Staatswissenschaftliche Untersuchungen und Erfahrungen, Zürich 1812, 1. Band

S. III.

522 Bände Hannover 1804. 
177) zu der Überzeugung, «daß die empirisch-rationale Heilkunde die strengsten Prüfungen der vernünftigen Kritik ebenso standhaft und siegreich aushalten kann als irgendeine empirische Wissenschaft und Kunst».

Die ganze Problematik, in welche die Medizingesetzgebung sogleich bei ihrer Entstehung durch die Auseinandersetzung mit Cabanis' Schrift geraten war, enthüllen die Staatswissenschaftlichen Untersuchungen und Erfahrungen über das Medicinalwesen nach seiner Verfassung, Gesetzgebung und Verwaltung, die der uns schon bekannte Јонаnм Stoll (1769-1848), Vetter zweiten Grades von Maximilian Stoll, 1812 in Zürich erscheinen ließ. Als er dies tat, stand er bereits neunzehn Jahre im Medizinaldienst, davon die letzten neun als Direktor des Medizinalkollegs von Westfalen, dessen öffentlichen Gesundheitsapparat er vollkommen neu und mit Erfolg aufgebaut hatte ${ }^{53}$. Die dabei gewonnenen Erfahrungen sowie seine Gedanken über eine allgemeine Gesundheitsgesetzgebung legt er in dem genannten Werke vor. Zu seiner Zeit hat es stärkste Beachtung gefunden, nicht zum letzten beim Altmeister des Faches selbst, bei Johann Peter Frank, welcher es an vielen Stellen der nach 1817 erschienenen Bände seiner Medizinischen Polizei zitiert ${ }^{54}$.

Vor die Behandlung seines eigentlichen Themas setzt Stoll - hierin Schmidtmann ähnlich - zwei Kapitel: «Zweifel gegen die Möglichkeit, Wirklichkeit und Nützlichkeit der medicinischen Doctrinen» (S. 3-38) und «Widerlegung der im vorhergehenden Capitel gegen die Arzneykunde aufgestellten Zweifel und Beschuldigungen» (S.39-86). Schon diese Anordnung in Argumentation und Gegenargumentation und noch mehr ihr Inhalt lassen unschwer das Vorbild erkennen, nämlich Cabanis' Schrift, die Stoll auch gleich S. 11 als seine Quelle zitiert. Dabei stellt er sich zur Aufgabe, die nach seiner Ansicht von Cabanis nicht erschöpfend behandelte Frage sowohl in negativer als in positiver Richtung entsprechend dem neuesten Stand der Medizin - seit der Abfassung von Cabanis' Schrift waren ja schon zweiundzwanzig Jahre verstrichen - weiterzuführen. Dies schien ihm im Hinblick auf sein Vorhaben, eine bestmögliche Medizinalverfassung vorzuschlagen, von besonderer Bedeutung, ja dringender Notwendigkeit. Denn worauf gründet sich diese Medizinalverfassung und Ge-

${ }^{53}$ J.STOLL, a. O. VIIff.

${ }^{54}$ J.P.FranK, a. O. 6A S.544.554; 6B 123.564.579ff.610 u. ö. und dies trotz der Auseinandersetzung in $6 \mathrm{~A}$ XVIII ff. 
setzgebung? Doch auf das vormundschaftliche Recht der Staatsregierung ${ }^{55}$, für das Wohl der einzelnen Staatsglieder zu sorgen. «Hieraus folgt aber auch, daß derjenige, der sich das Recht aneignet, für mich als Staatsglied zu sorgen, nicht nur in der Lage wirklich seyn müsse, diese Sorge besser, als ich Individuum selbst, übernehmen zu können, sondern auch verpflichtet sey, mich zu entschädigen, wenn ich durch die Befolgung seiner Vorschriften in Schaden komme. Der Gesetzgeber ... muß die Möglichkeit der medicinischen Gesetzgebung außer Zweifel setzen, das ist, beweisen - daß es über alle Gegenstände des körperlichen Wohlseyns objective Erfahrung giebt, ... ehe seine Entscheidungen die Gültigkeit von Gesetzen haben können.» In die uns vertraute Terminologie Cabanis' übersetzt heißt das: die vollkommene Gewißheit der Arzneiwissenschaft muß als Grundlage einer Gesundheitsgesetzgebung vorausgesetzt werden können.

Hier nun liegt der Grund, warum die von Cabanis entwickelte Problematik in unmittelbare Berührung mit der deutschen Staatsarzneilehre treten mußte, hier nun wird offenbar, warum die Medizinalpolitiker Deutschlands sich so brennend für die Schrift Cabanis' interessierten. Sind doch in ihr bereits alle die Fragen behandelt, die sich mit der Möglichkeit bzw. Unmöglichkeit objektiver Erkenntnisse in der Heilkunde und daraus ableitbarer sicherer Handlungen beschäftigen. Ja, mehr noch : in ihr findet sich bereits der Rang vorgezeichnet, den die Heilkunde dieser Zeit bei all ihrer Unvollkommenheit im Gefüge des Staatsorganismus noch einnehmen kann. Aber welch ein Rang ist das und welchen Nutzen kann diese Heilkunde dem Staate gewähren? Hier haben wir aus Cabanis' Lehren, die wir zu einem guten Teil überblickten, noch folgende Deduktion anzuführen:

Cabanis sieht die Notwendigkeit der Heilkunde in dem Bedürfnis der leidenden Menschen nach Linderung gegeben (C. $123 \mathrm{f}$.). Dieses Bedürfnis, so meint er, wird bleiben, 'selbst wenn man die Heilkunde austilgte. Der Erfolg davon müßte unfehlbar sein, daß eine größere Anzahl von Schlachtopfern in die Hände Unwissender fiele. Vom Arzt aber kann man erwarten, daß er viel Übles verhindert, selbst wenn er wenig Gutes stiftet; und wenn er selbst etwas Übles täte, so verhindert er doch noch ungleich mehr (C. 127).

Wir können es uns hier nicht versagen, darauf hinzuweisen, daß wenige Jahre später, nachdem Cabanis diese Gedanken niedergeschrieben hatte,

${ }^{55}$ J. StoLl, a. O. 6. In der Begründung der Haftpflicht des Staates bei eventuellen Mängeln der medizinischen Gesetzgebung folgt StoLl wörtlich J.B. Erhard, Theorie der Gesetze, Tübingen 1800, S. 5. 
ihre Wahrheit seinem Vaterlande Frankreich in drastischer Weise vor Augen geführt wurde. Wir meinen die Abschaffung sämtlicher ärztlicher Ausbildungsstätten durch das Dekret vom 18. August 1792 und die darauffolgende Anarchie im öffentlichen Gesundheitswesen Frankreichs ${ }^{56}$. Sie hat eindeutig bewiesen, daß es im Sinne Cabanis' auch einen «negativen Nutzen» der Heilkunde für den Staat gibt. Denn von einem solchen sprechen die Nachfolger Cabanis', wenn sie sich auf die eben dargelegte Deduktion beziehen.

Diese These Cabanis' vom negativen Nutzen der Heilkunde macht sich nun Stoll ganz zu eigen und wird hierin dem skeptischen Geist ${ }^{57}$ seines Vorbildes weitaus gerechter als beispielsweise sein so zuversichtlicher Vorgänger in der Bearbeitung des Stoffes, Schmidtmann. Stoll folgt dieser These, wenn er sagt ${ }^{58}$ : «So lange bis der Forschungsgeist der philosophischen Ärzte mehr erleuchteter Zeiten, die Arzneikunde als eine reine Wissenschaft begründen wird, muß man sich mit dem negativen Nutzen derselben, welcher als einzige Ausbeute aus den vorhergehenden Untersuchungen gegen allen Widerspruch wirklich gewonnen ist, begnügen.» Aus diesem Resultat, das ganz unmittelbar aus Cabanis übernommen ist, zieht der deutsche Staatsarzt die für den Charkater seiner Gesundheitsgesetzgebung so bedeutsame Folgerung ${ }^{59}$ : «Die zur Herbeiführung, Erhaltung und Beförderung des allgemeinen und privaten Gesundheitswohls der Staatsbürger abzweckenden Maßregeln müssen eine vorherrschend negative Richtung nehmen, d. i. die darauf sich beziehenden Vorschriften und Gesetze im Allgemeinen lehren und gebieten, was ... nicht geschehen soll ...»

Vielleicht charakterisiert nichts mehr den tiefen Unterschied zwischen französischer und deutscher Medizin dieser Epoche als die völlige Verschiedenheit der Wirkungen, die Cabanis' Schrift hier und dort ausgelöst hat: was dort zu einem neuen Anheben medizinischer Forschung, zur Entwicklung der experimentellen Methode in der Heilkunde führte, hat hier die Einsicht hinterlassen, daß Gesundheitsgesetze vorläufig nur negativ formuliert werden könnten. Ist diese Einsicht nicht auch zugleich ein sehr sprechendes Zeichen dafür, daß sich die Zeit der Krise voll bewußt war, in der die Medizin an der Schwelle des 19. Jahrhunderts stand?

${ }^{56}$ J.Stoll, a. O.82. E. Stüвler, Die französische Revolution und die Medizin, Sudh. Arch. 37 (1953), $136 \mathrm{f}$.

${ }^{57}$ Dagegen bezieht J. Stoll a. O. $13 \mathrm{ff}$. als Anhänger der Richtung Browns und Vertreter der KaNTschen Philosophie scharf Stellung gegen den Empirismus Cabanis'.

${ }^{58}$ J. Stoll, a. O.85.

${ }^{59}$ J. STOLL, a. O.85. 
Cabanis' Problematik von der Gewißheit der Arzneiwiossenschaft blieb weiter ein fester Bestandteil deutscher staatsarzneikundlicher Literatur und erhielt als solcher gleichsam seinen Stempel, als der Altmeister des Faches, Johann Peter Frank, sie in den 1817 erschienenen sechsten Band seiner Medizinischen Polizei aufnahm. Dort hat er sowohl der Exponierung als auch der Widerlegung der gegen die Heilkunde gerichteten Vorwürfe im 6. und 7. Paragraphen ${ }^{60}$ reichen Raum gewährt. Gleich anfangs zitiert er Cabanis teils im Original, teils in der Übersetzung von AYRER ${ }^{61}$. Mit großer Zustimmung folgt er dem französischen Arztdenker in seinem Grunddogma, daß Gesundheit sowohl als Krankheit empirische Zustände des Menschen und als solche beobachtbar und durch empirische Mittel veränderbar sind. Ebenso folgt er ihm auch in der agnostischen Haltung hinsichtlich der Ursachenlehre und stellt sachlich fest, daß die Heilkunde zwar keiner mathematischen Gewißheit fähig sei, deswegen aber doch sehr schätzbar bleibt.

Als Praktikus unterscheidet er drei Grade von Gewißheit bei der Diagnostik von Krankheiten ${ }^{62}$; nach diesen verschiedenen Graden richtet sich sein jeweiliges therapeutisches Handeln, wenn er sagt ${ }^{63}$ : «In einigen Krankheiten ist der Arzt freilich ungewiß, in andern ist er es weit weniger, und in vielen ist er es gar nicht. In der ersten Gattung hütet er sich gewissenhaft, nach thätigen, leicht nachtheiligen Mitteln zu greifen; und hier ist der Pazient wenigstens nicht übler daran, als wenn er trostlos, ohne Heilkünstler geblieben wäre. In dem zweyten Falle, verfährt der Arzt behutsam, lauschet oft der Natur beyzeiten ihr Geheimniß, ihre Winke ab, und befolget diese mit Gewandtheit, und je nachdem solche mehr oder weniger deutlich sind, mit mehr oder weniger Sicherheit ... In der dritten Gattung von Krankheiten befolget der Heilkünstler die bereits vortheilhaft gebrochene Bahn mit aller der Gewißheit, die menschlichen Handlungen gegeben ist, als welche bloß durch außerordentliche, sehr seltene Einwirkungen gestöret werden.»

So wie hier Frank auf dem festen Boden des Tatsächlichen und Praktischen steht, ebenso geht er auch in der Widerlegung der gegen die Heilkunde gerichteten Vorwürfe vor. Seine Argumentation weist - wir konnten

${ }^{60}$ J.P.Frank, System einer vollständigen medicinischen Polizey, Wien 1817, Band 6 A, S. 69-136.

${ }^{61}$ J.P. Frank, a.0.73ff.

${ }^{62}$ In der Unterscheidung dreier diagnostischer Gewißheitsgrade verhält sich FraNK gleich wie J. Köllner (vgl. S. 163f.), in ihrer Bewertung aber um vieles positiver als dieser.

${ }^{63}$ J.P.Frank, a.0.76ff. 
ähnliches schon bei Schmidtmann und Stoll vermerken - eine Fülle von neuen Zügen auf. Sie ist ihres philosophisch-abstrakten Charakters, der das Denken Cabanis' so kennzeichnet, entkleidet und arbeitet mit sehr gegenständlichen und konkreten Beweisen. Hier bekommen wir einen nicht uninteressanten Einblick, was Frank und seine Zeit als neue Errungenschaften der Heilkunde gewertet haben. Da nennt er zunächst in der prophylaktischen, seuchenabwehrenden Heilkunde die Einführung des salzsauren Gases durch Guyton de Morveau und die der salpetersauren Dämpfe durch James Carmichael Smyth ${ }^{64}$; dann kommt sofort Jenners große Entdeckung der Kuhpockenimpfung ${ }^{65}$, die Verhütung des Skorbuts ${ }^{66}$ und die Rettung der Scheintoten. Für die Chirurgie verzeichnet er u. a. als Fortschritt die Staroperation ${ }^{67}$, für die Geburtshilfe das konservative, naturgemäße Verfahren, für die Therapie u. a. die Einführung des roten Fingerhutes $^{68}$, um nur einige Beispiele für die so gegenständliche Art seiner Beweisführung zu geben.

Aber Frank gesteht auch frei, daß eine Reihe von Krankheiten, wie die Lungensucht, das Podagra, die Pellagra, bis jetzt unheilbar sind, aber doch auch gelindert werden können ${ }^{69}$.

In der hier vorgeführten Art hat Frank Cabanis' Problematik konkretisiert, hat ihre positive Beweisführung auf Grund von anerkannten Tatsachen verstärkt und sie nach dem neuesten Stand der Heilkunde erweitert. Seine Neuformung des Problems ist also bestimmt durch die zuversichtliche Haltung eines wohlgegründeten Empirismus, dem es fern liegt, aus dem gegebenen Wissensstande der Medizin irgendwelche negative Konsequenzen für die Gesetzgebung zu ziehen.

Als letztes Beispiel für die über Jahrzehnte reichende Wirkung Cabanis' auf die deutsche Staatsarzneikunde führen wir das 1819 erschienene Systematische Handbuch des Medicinal-Wesens des Wiener Hygienikers und Gerichtsmediziners Josef BerNT (1770-1842) ${ }^{70}$ an. Hier wird in den ersten zwölf Paragraphen der Einleitung ${ }^{71}$ die uns so wohlvertraute Problematik

64 J.P. Frank, a. O.96f.

65 J.P. Frank, a. $0.98 \mathrm{ff}$.

${ }^{66}$ J.P. Frank, a. $0.115 \mathrm{ff}$.

67 J.P.Frank, a. O.123.

68 J.P. Frank, a. O. 127.

${ }^{69}$ J.P.Frank, a. O.132f.

${ }^{70}$ Josef Bernt, Systematisches Handbuch des Medicinal-Wesens nach den k.k. österreichischen Medicinalgesetzen, Wien 1819.

${ }^{71}$ Josef Bernt, a. O. 1-5. 
Cabanis' abgehandelt. Wir erkennen die einzelnen Punkte seiner Argumentation genau wieder. Aber was ist hier aus der so geistvollen, beweglichen und lebendigen Darstellung des französischen Arztphilosophen geworden! Ihre Hauptpunkte sind in dürre Paragraphen aufgelöst und diese wieder nach der alphabetischen Unterabteilung säuberlich rubriziert. Von dem Geist aber, der diese Problematik geboren und getragen hat, ist in diesem Schema nichts mehr zu spüren. So ist Cabanis' Schrift Von dem Grade der Gewißheit der Arzneiwissenschaft, die ihre Zeit wie kaum eine andere erregt und befruchtet hat, nach einem Menschenalter in das Handbuchwissen der deutschen Staatsarzneikunde eingegangen.

\section{4}

Im vorhergehenden konnten wir zeigen, da $\beta$ die so dringlichen Erörte-

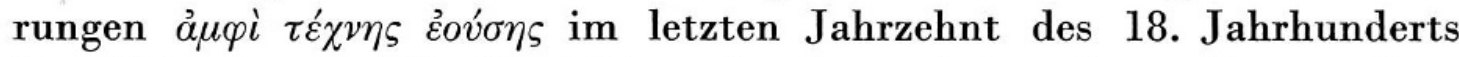
ihre Heimstätte bei den Nachfahren des französischen Sensualismus gefunden hatten und im Geist dieser Philosophie neugeformt wurden. Es bleibt uns noch übrig, in aller Kürze, ohne mehr als einen Anhang bieten zu wollen, darauf hinzuweisen, daß diesem französischen Vorgang ein analoger eigenständiger in Deutschland entspricht. Wir meinen jene Auseinandersetzung, die deutsche Ärzte unter dem Einfluß der Philosophie Kants ebenfalls im letzten Jahrzehnt des 18. Jahrhunderts über die Grundlagen der Medizin führten. Sie konzentrierte sich auf eine Fragestellung, die ohne weiteres in der Formulierung ihre Herkunft aus Kantschem Denken verrät: Ist Heilkunde als Wissenschaft möglich, ist sie wirklich und wie ist sie es ?

Unter diesen und ähnlichen Titeln erörterten zahlreiche von Kant beeinflußte Ärzte in verschiedenen Aufsätzen, für die ihnen Röschlaubs Magazin zur Vervollkommnung der theoretischen und praktischen Heilkunde ${ }^{72}$ den Raum bot, die denkmethodischen Voraussetzungen der Medizin. Bevor wir aber diese Aufsätze nennen, haben wir auf eine Auseinandersetzung gleichen Inhaltes aufmerksam zu machen, die in Form eines Angriffes auf die Medizin erfolgte und schon zufolge ihres weithin wirkenden Publikationsortes bei Ärzten und Laien des damaligen Deutschlands größtes Aufsehen erregte $^{73}$. Es ist der Aufsatz «Über die Medicin. Arkesilas an Ekdemus», der anonym im 2. Bande Der Neue Teutsche Merkur 1795 erschien ${ }^{74}$, einem

${ }^{72}$ Vgl. das Programm des Magazins in Röschlaubs Bemerkungen 1 (1799), 4f.

${ }^{73}$ Vgl. die diesbezüglichen Bemerkungen J.P.Franks, a. 0.63; ferner Salzb. medic.-chir. Zeitung 2 (1800), 78-80.

${ }^{74}$ Dort S. 337-78. 
Organ, das bekanntlich Wieland redigierte. Dem Skeptiker Arkesilas werden hier alle Zweifel supponiert, die der Kant-Schüler und damalige Nürnberger Arzt Johann Benjamin Erhard (1766-1827) - denn er bekennt sich später als Verfasser dieser Invektive ${ }^{75}$ - in scharfer Ironie an den bisher üblichen Methoden ärztlichen Erkennens und Handelns äußert ${ }^{76}$. Dieser Aufsatz wurde gleichsam zum Programm, «Arkesilas» das Stichwort $^{77}$, unter dem die Skepsis in der Medizin in Deutschland ihr Haupt erhob. Daran änderte auch nichts die scharfe Widerlegung und Abweisung, die Erhards Angriff sogleich erfuhr. Sie richtete sich nicht weniger gegen Inhalt und Form als auch gegen den Erscheinungsort der Invektive, den Teutschen Merkur, traf somit seinen Herausgeber Wieland selbst. Diese Entgegnung stammt von keinem geringeren als Christoph Wilhelm HufELAND (1762-1836) und verleiht dadurch allein schon der eben angegangenen Auseinandersetzung Gewicht. Sie erschien im selben Band des Teutschen Merkur zugleich mit einer Rechtfertigung Wielands ${ }^{78}$. In weitaus sachlicherem Ton ist der Artikel Erhards «Über die Möglichkeit der Heilkunst» gehalten, den er im 1. Band von Röschlaubs Magazin zur Vervollkommnung der theoretischen und praktischen Heilkunde $1799^{79}$ erscheinen ließ. Hier geht es darum, an dem durch Kant gereinigten Erfahrungsbegriff das vielfach Trügerische ärztlicher Schlüsse aufzuzeigen und als Grundlage wahren ärztlichen Erkennens die «objektive Erfahrung» zu setzen, d.i. «die Gewißheit, das zwischen bestimmten Erscheinungen ein Kausalverhältnis ist».

Mit einer im wesentlichen gleichen Thematik beschäftigt sich der Aufsatz von GeIER, «Analytik des Begriffes der Heilkunst» ${ }^{80}$. Er versucht die Möglichkeit und Notwendigkeit der Heilkunde a priori darzutun und kommt zur Überzeugung, daß ihr der nämliche Grad von Gewißheit und Zuver-

${ }^{75}$ Röschlaubs Magazin zur Vervollkommnung der theoretischen und praktischen Heilkunde 2 (1799), $7 \mathrm{ff}$.

${ }^{76}$ Es werden im wesentlichen dieselben Einwände gegen die Heilkunde vorgetragen wie bei Cabanis, ohne daß eine literarische Abhängigkeit stattgefunden haben kann. Denn Cabanis' Schrift erschien ja erst 1798 im Druck.

${ }^{77}$ Röschlaubs Magazin zur Vervollkommnung der theoretischen und praktischen Heilkunde 1 (1799), 227; 2 (1799), 7.

${ }^{78}$ Chr. W. Hufeland, Ein Wort über den Angriff der razionellen Medicin im Neuen Teutschen Merkur, August 1795, Der Neue Teutsche Merkur 2 (1795), 138-55.

${ }^{79}$ Dort S. 23-86 und in kürzerer Zusammenfassung in J.B. Erhard, Theorie der Gesetze, Tübingen 1800, S. 10-7.

${ }^{80}$ Röschlaubs Magazin zur Vervollkommnung der theoretischen und praktischen Heilkunde 1 (1799), 257-78. 
lässigkeit zuzuerkennen ist, den alle Erfahrungswissenschaften besitzen. Dieses zweite Untersuchungsergebnis zeigt, daß es den Ärzten in der Nachfolge Kants bei ihren wissenschafts-theoretischen Analysen letztlich um das gleiche Anliegen ging wie dem französischen Arztdenker: den Gewißheitsund Zuverlässigkeitsgrad medizinischer Methoden zu bestimmen.

Besonders klar wird dies in einem dritten Aufsatze desselben Magazins von J. KöLlner, der wieder den signifikanten Titel trägt: «Ist die Heilkunde als Wissenschaft betrachtet möglich und wie ist sie es ?» ${ }^{81}$ Hier wird für die einzelnen Disziplinen der Medizin, Physiologie, Pathologie, Semeiotik, Materia medica, allgemeine und spezielle Heilkunde, in einer für die damalige Struktur dieser Fächer sehr aufschlußreichen Weise gezeigt, daß sie auf Erfahrungsgrundsätzen von komparativer Allgemeinheit beruhen, daß somit ihren Ergebnissen im besten Falle Wahrscheinlichkeit zukommen kann. Anschließend wird eine Zergliederung des Verfahrens ärztlicher Erkenntnisfindung gegeben. Dabei wird das Ineinandergreifen von analytischen, synthetischen, induktiven und deduktiven Denkprozessen in einer Weise geschildert, die vielfach an das vierfache Methodengefüge bei M. Hartmann ${ }^{82}$ erinnert. Leitende Ideen werden im Sinne Kants postuliert, sofern der Medizin wissenschaftlicher Systemcharakter zukommen soll. Doch dürfen diese Ideen niemals konstitutiv = setzend sein, sondern müssen immer nur regulativ = leitend bleiben. Diese Forderung enthüllt auch den Grund für die enge Verbindung, die wir bei all den genannten kantischen Ärzten, wie schon S. 156 vermerkt, mit dem Brownschen System beobachten. Sie meinten nämlich, in Browns Begriff der Erregbarkeit eine solch leitende Idee gefunden zu haben.

Dadurch ist auch ein früheres Werk des bereits öfter genannten JoHANN SтоLL, Versuch einer medicinischen Beobachtungskunst (Zürich 1802), charakterisiert. In ihm versucht Stoll, vom Kantschen Erfahrungsbegriff ausgehend, eine ärztliche Methodologie aufzubauen, verstrickt sich aber gerade dadurch, daß er die Erregbarkeit als leitende Idee anerkennt, in die Netze des Brownianismus. So können wir seine Haltung als typisch für diesen Kreis kantischer Ärzte bezeichnen, die trotz aller Bemühungen, einen geläuterten Erfahrungsbegriff in das ärztliche Denken einzuführen, letzten Endes selbst systemgläubig im Sinne des Brownianismus wurden.

81 Ebendort, S. 303-87.

${ }^{82}$ M. HartmanN, Die philosophischen Grundlagen der Naturwissenschaften, Jena 1948, S. $121 \mathrm{ff}$. 
Andreas Röschlaub selbst (1768-1835), damals Kliniker in Landshut und Hauptführer des Brownianismus in Deutschland, ist aus den eben geschilderten Voraussetzungen heraus zu verstehen. Bei ihm tritt ein anderer Zug, der all diesen Kritikern des Erfahrungsbegriffes gemeinsam ist, besonders stark in den Vordergrund. Wir meinen die skeptische Haltung gegenüber einer Medizin, deren Wissenschaftscharakter im Sinne Kants noch lange nicht erwiesen ist. Röschlaub ist es nun, bei dem diese Skepsis eine Wirkung zeitigt, die im Zusammenhange mit den im 3. Abschnitt erörterten Problemen von besonderem Interesse ist.

In seinem Buch Über Medizin ihr Verhältni $\beta$ zur Chirurgie nebst Materialien zu einem Entwurfe der Polizei der Medizin (Frankfurt am Main 1802) beschäftigt er sich, wie wir dies als zeittypisch für andere Ärzte zeigen konnten, mit dem Entwurf zu einer Medizinalverfassung und Verwaltung. Bevor er seine, dann durchaus positiven Vorschläge bringt, diskutiert er aber eingehend (S. 24-62), ob man bei der gegebenen Unvollkommenheit der Medizin als Wissenschaft eine solche Verfassung verantworten könne. Diese Erörterung, ob «denn die Gesundheitspflege und jeder der Zweige derselben, Hygiene und Medizin, wirklich gegeben» seien (S. 24), führt der Herausgeber des Magazins zur Vervollkommnung der Heilkunde ganz in dem skeptischen Sinne der in diesem Organ veröffentlichten und oben genannten Aufsätze. Ebenso nimmt er hier Gedankengänge auf und profiliert sie schärfer, die bereits Joh. Benj. Erhard in seiner 1800 in Tübingen erschienenen Theorie der Gesetze (S. $6 \mathrm{ff}$.) geäußert hat. Damit ist die durch die Kantsche Philosophie bestimmte Auseinandersetzung über die Grundlagen der Medizin bei Röschlaub in gleicher Weise zum Ausgangspunkt und zur Voraussetzung für gesundheitspolitische Maßnahmen geworden, wie wir dies im vorigen Abschnitt für eine Reihe staatsarzneikundlicher Werke im Zusammenhange mit jener Problematik zeigen konnten, die von Cabanis ausging.

Wir können hier auf eine genauere Analyse des angezogenen Abschnittes bei Röschlaub, so reizvoll dies wäre, nicht mehr eingehen; möchten aber doch bemerken, daß die ganze Art seiner Argumentation nicht auf gleichzeitige Beeinflussung durch Cabanis schließen läßt, den er auch nirgends erwähnt. Anders ist dies bei STOLLS Staatswissenschaftlichen Untersuchungen und Erfahrungen, der, wie wir S. 162 zeigen konnten, in seiner skeptischen Haltung und der daraus resultierenden Beschränkung in der Medizinalgesetzgebung ausdrücklich Cabanis folgt. Hier haben wir noch ergänzend hinzuzufügen, daß ihm diese Nachfolge Cabanis' leicht gemacht 
wurde, weil sie sich bei ihm mit der gleichen Grundhaltung der kantischen Ärzte verbindet ${ }^{83}$. Die Art dieser Verbindung im einzelnen zu analysieren, kann nicht mehr Aufgabe dieses Abschnittes sein.

Wohl aber haben wir uns zum Schluß noch mit einem Werke zu beschäftigen, das den Mainzer Kliniker Georg von WedeKIND (1761-1831) zum Verfasser hat und Über den Werth der Heilkunde (Darmstadt 1812) handelt. Es ist eine Apologie der Medizin, 1808 geschrieben ${ }^{84}$ von einem erfolgreichen und angesehenen Praktiker der damaligen Zeit, der sich nach einer dreißigjährigen Erfahrung als Hofarzt, akademischer Lehrer, als Physikatsund Armeearzt zu einem wohlausgewogenen Eklektizismus bekennt ${ }^{85}$. Nun hat er sich - wie so viele Ärzte dieser Zeit - die Aufgabe gestellt, «für den Staatsmann ein brauchbares Buch über die Polizei der Medizin» auszuarbeiten. Es ist nun für unsere ganze Problematik höchst aufschlußreich zu erfahren, welches Motiv er mit diesem Vorhaben verbindet. In der Vorrede ${ }^{86}$ sagt er es uns selbst: «Er (sc. der Verfasser) hielt es aber für nöthig dieser litterarischen Arbeit eine Einleitung vorauszuschicken, worin im allgemeinen das Verhältnis der Medizin zur Politik bestimmt würde. Existiert denn auch würklich eine Heilkunde, ist sie möglich, stiftet sie überwiegenden Nutzen? Diese sogar von Ärzten aufgestellten Fragen erregen izt zu große Sensation und müssen erörtert werden, bevor sich angeben läßt, wie, und inwieweit, die Regierungen sich mit der Medizin einlassen dürfen ...»

Hier sind wir mitten in der uns schon wohlbekannten Problematik der Zeit: Erörterung der Grundlagen der Medizin als Voraussetzung einer medizinischen Gesetzgebung, die auf dem Vormundschaftsrecht des absolutistischen Staates beruht. Während aber in den entsprechenden Werken von Erhard, Röschlaub, Schmidtmann und Stoll diese Erörterung ein bis zwei Kapitel beansprucht, ist sie hier - ursprünglich nur als Einleitung gedacht - über das eigentliche Vorhaben, eine medizinische Polizei auszuarbeiten, zu einem selbständigen Werk von 381 Seiten angewachsen. So groß war also das Bedürfnis, sich mit Fragen auseinanderzusetzen, deren Herkunft die von Wedekind benutzte Terminologie in der ausgeschriebenen Stelle sofort verrät: es ist der Ärztekreis um Röschlaub und Erhard, die Wedekind nachgerade zu den Gegnern der Heilkunde zählt, wenn er fest-

${ }^{83}$ J.SToLL, Versuch einer medicinischen Beobachtungskunst, Zürich 1812, S. IIf.

${ }^{84}$ G.v. WeDEKIND, a. O.X.

${ }^{85}$ G.v. WEDEKIND, a. $0.318 \mathrm{ff}$.

${ }^{86}$ G.v. WeDEKIND, a. O.VIIf. 
stellt ${ }^{87}$ : «Auch ist es wohl nicht zu läugnen, daß die Gegner der Medizin sich vermehrten, seitdem die Kantische Philosophie eine strengere Prüfung des Gewissen in unserer Erkenntniß, zumahl des Inhalts der Erfahrungswissenschaften, veranlaßt hatte. Ja es scheint, daß schon die Behauptung, es gehöre die Medizin nicht mit zu den eigentlichen Wissenschaften, bei vielen das Zutrauen auf ihre Zuverlässigkeit geschwächt hätte...» «Und was mußte man dazu sagen, wenn ein Röschlaub seine Zweifel an der Möglichkeit und seinen Unglauben an der Würklichkeit der Arzneiwissenschaft öffentlich mittheilte ${ }^{88}$, dabei aber auf eine strenge Polizei der Medizin, auf Besoldung der Ärzte und auf Begünstigung der medizinischen Lehranstalten antrug ${ }^{89}$ ?»

Aus den angeführten Stellen geht klar hervor, daß sich Wedekinds Apologie der Heilkunde vor allem gegen die aus dem kantischen Ärztekreis erwachsene Problemstellung richtet, die auch die Anordnung seiner ganzen Polemik bestimmt, wie: «Beweis von der Möglichkeit der ärztlichen Gesundheitspflege, Widerlegung der Gründe gegen die Würklichkeit der Gesundheitspflege» usw. Eine glatte Absage an die kantischen Ärzte bedeutet die Stelle S. 118: «Über die Frage: ob die Medizin eine Wissenschaft sey? will ich nicht streiten. Die Untersuchung, ob sich ihr Inhalt aus einem allgemeinen Prinzip herleiten, oder auf ein solches hinleiten lasse, mag ferner jungen, oder müßigen Ärzten überlassen bleiben. An diesem perpetuum mobile kann ich nicht arbeiten.»

Doch glauben wir aus einigen Partien von Wedekinds Beweisführung ${ }^{90}$ auch den Einfluß Cabanis' durchzuspüren, den er allerdings nie nennt. Jedenfalls ist es wahrscheinlich, daß Wedekind bei seiner intensiven Berührung mit der französischen Medizin, für die allein schon der ausführliche Bericht über die französische Medizinaleinrichtung zeugt ${ }^{91}$, auch mit der ihre Zeit so erregenden Schrift Cabanis' bekannt war. Ist diese Vermutung richtig, dann wäre für Wedekind ein Verhalten anzunehmen, das dem Stolls

${ }^{37}$ G.v. WEDEKIND, a. O. 4 .

${ }^{88}$ Eben in der von uns angezogenen Partie Über Medicin ihr Verhältniß zur Chirurgie... (Frankfurt am Main 1802), S. 24-62.

${ }^{89}$ Dies war der Inhalt von Röschlaubs genanntem Werk. Die Inkonsequenz seines Vorgehens kritisiert in gleicher Weise wie WEDEKIND auch J. STOLL, Staatswissenschaftliche Untersuchungen ... S.9, und J.P.Frank, a.0.64.

${ }^{90}$ Vgl. u.a. die Partie über die Entstehung der Heilkunde bei Wedekind, a.0.96f., jene über die empirische Gewißheit S. 101.

${ }^{91}$ G. v. WEDEKIND, a. 0.7-34. 
entgegengesetzt ist: während Stoll der Skepsis Cabanis' folgte, seinen Empirismus aber ablehnt ${ }^{92}$, hätte Wedekind bei seiner Verteidigung der Medizin, ohne die Skepsis des Franzosen zu berücksichtigen, Anregungen aufgenommen, die ihm aus dessen empirischer Haltung zukamen.

Auf alle fruchtbaren Erkenntnisse einzugehen, die Wedekind im geistigen Ringen um die Grundlagen der Medizin erwuchsen, ist hier nicht mehr der Raum. Ein einziger Satz sei ausgeschrieben, der in seiner monströsen Form einen Gedanken von grundsätzlicher Wichtigkeit birgt ${ }^{93}$ : «Solche Untersuchungen führen aber ebenfalls zu sichern Schlüssen, wenn wir den todten mit dem lebendigen Körper nur in so weit vergleichen, als beide übereinkommende Erscheinungen geben können, und wenn wir demnächst zusehen, ob die aus den Erfahrungen am todten Körper gezogenen Schlüsse mit denen, welche sich aus den, am lebendigen gemachten Erfahrungen ergeben, nicht in Widerspruch stehen, sondern, ob sie sich vielmehr wechselseitig aufhellen?» Was WEDEKIND hier formuliert, ist nicht mehr und nicht weniger als das Programm der zweiten Wiener Schule, wie Rokitansky und SKoDA es verwirklicht haben.

${ }_{92}$ J. StоLL, Staatswissenschaftliche Untersuchungen ... $13 \mathrm{f}$.

${ }^{93}$ G.v. WEDEKIND, a. O.115. 